\title{
Theoretical Aspects of Energy Confinement in Spheromaks
}

T. K. Fowler

This paper was prepared for submittal to the

Workshop on Establishing the Physics Basis Needed to Access

the Potential of Compact Toroidal Reactors

Oak Ridge National Laboratory

Oak Ridge, TN

July 19-21, 1994

November 16, 1994

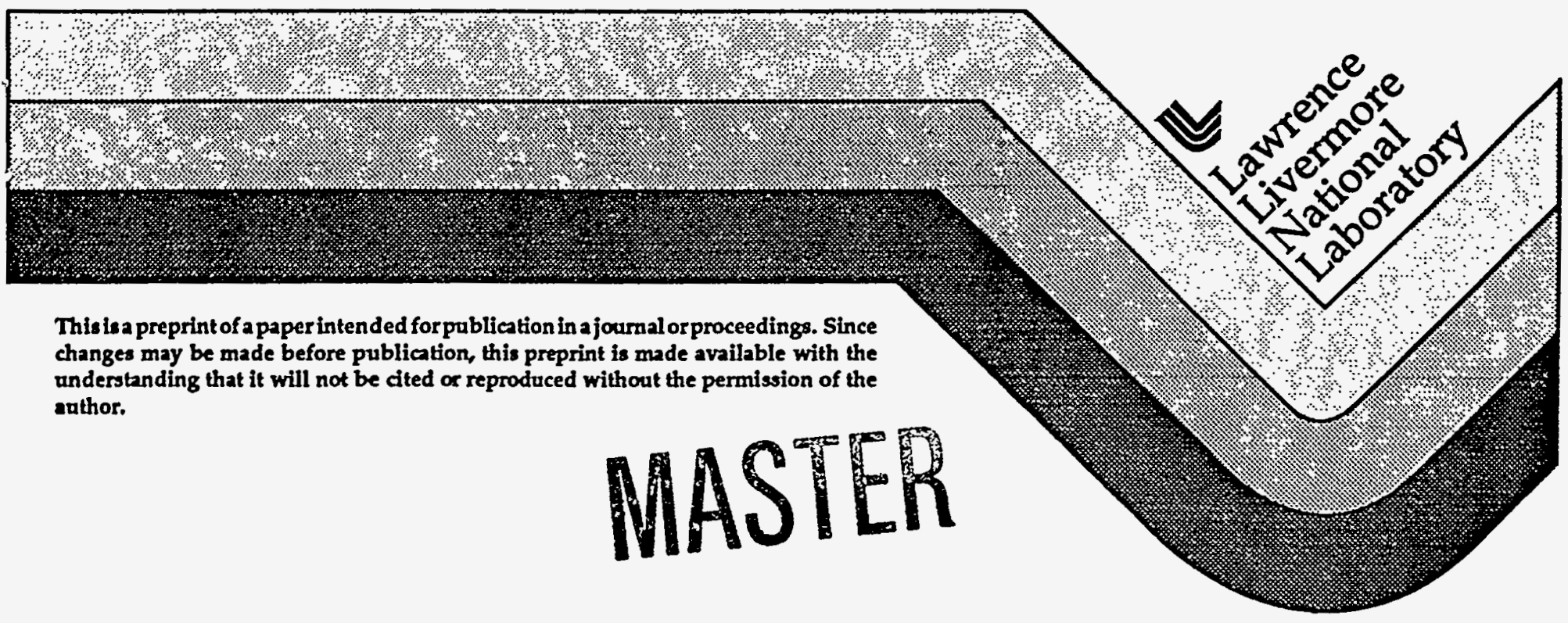




\section{DISCLAIMER}

This report was prepared as an account of work sponsored by an agency of the United States Government. Neither the United States Government nor any agency thereof, nor any of their employees, make any warranty, express or implied, or assumes any legal liability or responsibility for the accuracy, completeness, or usefulness of any information, apparatus, product, or process disclosed, or represents that its use would not infringe privately owned rights. Reference herein to any specific commercial product, process, or service by trade name, trademark, manufacturer, or otherwise does not necessarily constitute or imply its endorsement, recommendation, or favoring by the United States Government or any agency thereof. The views and opinions of authors expressed herein do not necessarily state or reflect those of the United States Government or any agency thereof. 


\section{DISCLAIMER}

Portions of this document may be illegible in electronic image products. Images are produced from the best available original document. 


\title{
Theoretical Aspects of Energy Confinement in Spheromaks*
}

\author{
T. K. Fowler
}

\begin{abstract}
It is shown that, despite the poor global energy confinement observed in spheromak experiments to date, the long-term prospects may be favorable as spheromaks are scaled to larger size and higher temperatures. The present performance is traced to excessive magnetic energy loss at the edge compared to tokamaks and heat transport due to magnetic fluctuations, both of which should scale away as the temperature increases.
\end{abstract}

\section{Introduction}

In a recent paper, ${ }^{1}$ we have argued that heat confinement in the core of a spheromak may be good despite the poor global energy confinement time exhibited in experiments to date. ${ }^{2}$ Here we examine further the core confinement itself, taking into account heat transport due to magnetic turbulence. Both the degraded global energy confinement and the turbulent heat transport arise from the tendency of the spheromak to maintain a Taylor state. $^{3}$

We first review the argument that maintaining a Taylor state degraded global energy confinement in previous spheromak experiments, all of which employ only ohmic heating. In a Taylor state, the current density $j$ is nearly constant, independent of position. Then, since the resistivity $\eta$ is largest at the edge where temperatures are low, the ohmic heating, $\eta j^{2}$, is largest at the edge where it does no good in heating the core. Contrast this with an ohmically-heated tokamak in which $E=\eta j$ is constant; $j$ decreases as $\eta$ increases; and hence $\eta j^{2}=E j$ decreases at the edge so that most of the heating occurs in the core. For the tokamak, a global energy confinement time obtained by dividing the stored kinetic energy by the ohmic power gives

*This work performed under the auspices of the U.S. Department of Energy by the Lawrence Livermore National Laboratory under Contract No. W-7405-ENG-48. 
a meaningful measure of energy confinement in the core. But for the spheromak, this would be a meaningless figure of merit, since most of the ohmic power is wasted in maintaining a Taylor state. Of course, if the core confinement itself is good, none of this matters in the long run. Indeed, in Ref. 1 it is argued that, despite the inefficiency of ohmic heating in spheromaks, the core confinement is already good enough to scale to ohmic ignition in a device of moderate size. 1

That this picture is consistent with the data is shown in Fig. 1, which compares the theory with experimental measurements of the magnetic decay time, $t_{w}$, from Ref. 2. The theoretical curve is a plot of the following formula, derived in Ref. 1 for a Taylor current profile and a parabolic electron temperature profile as is typically observed ${ }^{1}$

$$
\left(t_{\mathrm{W}}\right)_{\text {theory }}=0.5 \mathrm{R}^{2} \mathrm{~T} \sqrt{\mathrm{T}_{\mathrm{E}}}
$$

Here $R$ is the flux conserver radius in meters, $T$ is the core temperature in $\mathrm{keV}$ and $\mathrm{T}_{\mathrm{E}}$ is the edge temperature. Note the linear dependence on $\mathrm{T}$ in Eq. 1, which may explain the nearly-linear dependence of the measured $t_{w}$, $\mathrm{T}_{\mathrm{E}}$ being relatively constant. It is this dependence on the edge temperature that reflects the waste of ohmic power at the edge (up to 90\%). For a tokamak, we expect $t_{w} \propto T^{3 / 2}$, not $T$.

Figure 2 plots the global energy confinement time obtained if we simply divide the kinetic energy by the ohmic power expressed in terms of $t_{W}$. This is given by 2

$$
\left(\tau_{\mathrm{E}}\right)_{\text {global }}=\frac{3}{2}<\beta>t_{\mathrm{w}}
$$

where $\langle\beta\rangle$ is the volume average of the pressure parameter $\beta$ as usually defined. Note that, since $\langle\beta>$ turns out to be relatively constant for this data, like $t_{w}$ itself the global energy confinement time thus defined increases with electron temperature, as classical processes do. However, according to our thesis, we should take no comfort in this seemingly favorable scaling with 
temperature, since it may have nothing to do with the actual heat confinement in the core.

\section{Heat Transport Due to Helicity Propagation}

The tendency of $\langle\beta>$ to reach a limiting value in spheromaks, mentioned above, may be yet another artifact of the tendency toward a Taylor state. Here we exclude those cases in which a specific pressure-driven instability sets such a limit. 4

The possibility of a limiting $\langle\beta\rangle$ is associated with the propagation of helicity by magnetic fluctuations and heat transport due to these fluctuations. Helicity (or current) propagation, required to maintain the Taylor state, follows from the time-averaged ohm's law

$$
\vec{E}_{0}=-\langle\tilde{v} \times \tilde{\beta}\rangle+\eta \overrightarrow{j_{0}}
$$

In spheromaks, $\overrightarrow{\mathrm{E}}_{0}=0$ on closed field lines so that the only means of generating current is the "dynamo" term, $\langle\tilde{v} \times \tilde{\beta}\rangle$, where $\tilde{B}$ is the magnetic fluctuation and $\tilde{v}$ is the fluid velocity perturbation related to $\tilde{B}$ by $\tilde{v} \propto \gamma \tilde{\mathbf{B}}$, where $\gamma$ is a growth constant. Following Bhattacharjee and Hameiri, 5 we can use Eq. (3) and Faraday's law to construct a field energy equation,

$$
\frac{\partial}{\partial t} \frac{B^{2}}{2 \mu_{0}}+\frac{1}{r} \frac{\partial}{\partial r}(r P(r))=-\eta j^{2}
$$

where $\mathrm{P}(\mathrm{r})$ is a Poynting vector, derived from the "dynamo" term, which can be written as

$$
\begin{aligned}
& \mathrm{P}(\mathrm{r})=\left\langle\mathrm{X} v_{\mathrm{A}} \frac{\tilde{\mathrm{B}}^{2}}{\mu_{0}}\right\rangle \\
& \mathrm{X}=\left(\gamma \tau_{\mathrm{A}}\right)
\end{aligned}
$$


where $v_{A}$ is the Alfven speed and $\tau_{A}=a / v_{A}$, a being the plasma minor radius of order $a \simeq R / 2$. We can also write an electron heat flow equation,

$$
\frac{\partial}{\partial t}\left(\frac{3}{2} n T\right)+\frac{1}{r} \frac{\partial}{\partial r} \operatorname{rn} \chi \frac{\partial T}{\partial r}=\eta j^{2}
$$

where we take $\chi$ to be the Rechester-Rosenbluth thermal diffusivity due to parallel heat flow in a turbulent magnetic field, 6

$$
\chi=v_{\mathrm{e}} \mathrm{L}_{\mathrm{c}} \frac{\tilde{\mathrm{B}}^{2}}{\mathrm{~B}^{2}}
$$

Here $v_{e}$ is the electron thermal speed and $L_{c}$ is a correlation length of order $R$ in the collisionless regime.

For steady state (zero time derivatives), adding Eqs. (4) and (7) gives

$$
-\mathrm{n} \chi \frac{\partial \mathrm{T}}{\partial \mathrm{r}}=\mathrm{P}(\mathrm{r})
$$

Then, using $v_{e}=\beta^{1 / 2}\left(m_{i} / m_{e}\right)^{1 / 2} v_{A}$ and $\beta=n T \mu_{0} / B^{2}$, combining Eqs. (5), (8) and (9) gives

$$
\beta=\left(\frac{m_{e}}{m_{i}}\right)^{1 / 3}\left(\frac{R X}{L_{c}}\right)^{2 / 3}
$$

More properly, this is the maximum $\beta$ achievable by ohmic heating since any additional contribution to $\chi$, due to collisions, etc., would only reduce $\beta$.

\section{Fluctuation Levels}

The equations in the previous section can be used to estimate the fluctuation level $\tilde{\mathrm{B}}$, and from this the thermal diffusivity $\chi$. Again assuming steady-state, integrating Eq. (4) and using Eq. (5) gives roughly 


$$
\mathrm{X} \frac{\tilde{\mathrm{B}}^{2}}{\mathrm{~B}^{2}}=\frac{1}{\mathrm{~S}}
$$

where $\mathrm{S}$ is the magnetic Reynolds number, .

$$
\mathrm{S}=\frac{\mathrm{a}^{2} \mu_{0}}{\eta} \frac{1}{\tau_{\mathrm{A}}}
$$

Assuming that resistive tearing modes are responsible for magnetic energy (and helicity) propagation, Strauss finds a non-linear (hyper-resistive) growth constant $\gamma \sim \tau_{A}^{-1}(\tilde{B} / B)$, whereupon, by Eq. (6), $X=\tilde{B} / B$, and by Eq. (11),?

$$
\frac{\tilde{\mathrm{B}}}{\mathrm{B}}=\mathrm{S}^{-1 / 3} \text { Hyper-resistive }
$$

On the other hand, comparing Eq. (10) to experimental values of $\beta$ in the collisionless regime suggests $X$ of order unity for $L_{c}=R$; or by Eq. (6), $\gamma \sim \tau_{A}^{-1}$, indicative of Alfven waves. Then, with $\mathrm{X}=1, \mathrm{Eq}$. (11) gives

$$
\frac{\widetilde{\mathrm{B}}}{\mathrm{B}}=\mathrm{S}^{-1 / 2} \cdot \quad \text { Alfven }
$$

Connor and Taylor 8 obtain this same scaling for resistive MHD phenomena, but only for radial wavelengths $\ll$ a; and some reversed-field pinch data exhibits $S^{-1 / 2}$ scaling. 9

Both scalings, Eqs. (13) and (14), indicate a sharp reduction in fluctuation levels and $\chi$ as the temperature increases and $\eta$ decreases. Assuming Eq. (8) to be correct, the more favorable Alfven scaling appears to be in better accord with the experimentally observed values of $\beta$ (discussed above) and temperature (see Ref. 1).10 However, as we shall see in the next section, for either scaling magnefic turbulence due to helicity propagation is unlikely to be the dominant source of heat transport in future spheromak experiments at high temperatures. 


\section{Scaling}

In Ref. 1, scaling laws are presented for ohmically-heated spheromaks giving $T$ as a function of $B$ and $R$ for different functional forms of $\chi$. Assuming quasi-steady state, $\mathrm{T}$ is obtained by integrating Eq. (7) over the core volume neglecting the time-derivative term. As explained in Ref. 1 , the density is set equal to the minimum value to avoid beam-driven instability, given by $n v_{i}=$ $j$ where $v_{i}$ is the ion thermal speed. The result for different scalings is 1

$$
\begin{array}{ll}
\mathrm{T}=\mathrm{K} \mathrm{B} \mathrm{R} \mathrm{R}^{3 / 4} & \text { Hyper-resistive } \\
\mathrm{T}=\mathrm{KB} \mathrm{B}^{2} \mathrm{R}^{2} & \text { Alfven }
\end{array}
$$

and for gyrobohm scaling $\left(\chi \propto \mathrm{T}^{3 / 2} / \mathrm{B}^{2} \mathrm{R}\right)$

$$
\mathrm{T}=\mathrm{K} \mathrm{R}^{6 / 7} \mathrm{R}^{4 / 7} \quad \text { Gyrobohm }
$$

Choosing $\mathrm{K}$ values to fit existing experimental data provides the scaling to future experiments. As concluded in Ref. 1, gyrobohm is the most pessimistic among these three scalings, due to its unfavorable dependence on temperature. Hence, assuming gyrobohm scaling to design future experiments would be a conservative choice. On the other hand, this may actually be too pessimistic. As already mentioned, the limiting values of beta observed may be better explained by core heat transport due to magnetic turbulence with Alfven scaling, implying that gyrobohm transport, if present, was too weak to dominate. Alfven scaling, not given explicitly in Ref. 1, is the most optimistic among the three scalings discussed here.

\section{Discussion}

We have suggested that, regarding heat confinement, the prospects for spheromak fusion reactors may have been under-appreciated on two counts. 
First, the usual global energy confinement time useful in evaluating tokamak performance is misleading when applied to the ohmically-heated spheromak data available to date, because in spheromaks ohmic heating is largely wasted in the edge. This hypothesis could be tested by direct heating of the core by auxiliary power (within the limits set by pressure-driven modes), and by scaling ohmic-heating experiments to the larger size and field strength needed to reach high temperatures by ohmic heating.

Secondly, concerns about heat transport due to magnetic turbulence inherent in maintaining the Taylor state may be unfounded. While magnetic turbulent transport may in fact be the dominant mechanism in the small, low-temperature experiments of the past, turbulent fluctuation levels should decrease markedly as collisional resistance decreases at higher temperatures.

These claims touch upon the oldest debate in fusion research; namely, does success require external control to maintain a stable plasma, or can the plasma be trusted to find its own stable state. That a self-stabilized reactor would be simpler and cheaper is reasonably certain. 11 That the simplest of toroidal concepts, the spheromak, possesses a stable state - the Taylor state - is also reasonably certain, both theoretically and experimentally. ${ }^{3}$ The remaining question, whether the spheromak automatically seeks this stable state in a non-destructive manner, is, if less certain, sufficiently compelling to warrant continued research on spheromaks.

The question of non-destructive self-stabilization reduces to knowing what destroys the magnetic field, since potentially destructive magnetic fluctuations need only grow to the level required to propagate current from one region to another at the rate needed to restore the decaying Taylor current profile. Here again we can assert the main point with reasonable certainty; namely, magnetic fields almost always decay not anomalously but by classical or neoclassical resistivity, a remarkable but theoretically plausible 12 fact now demonstrated in tokamaks all the way to the temperatures required for fusion reactors. It is this latter point, expressed via the ohmic decay term on the right hand side of Eq. (4), that justifies the 
conclusion that fluctuations and transport should diminish at high temperature, more or less independently of the exact form of the Poynting vector.

Though not new, the relevance of these points as a motivation for further research on spheromaks has perhaps not been sufficiently emphasized. 


\section{References}

1. T. K. Fowler, J. S. Hardwick, and T. R. Jarboe, comments Plasma Phys. Controlled Fusioin, 16, 91 (1994).

2. F. J. Wysocki, J. C. Fernandez, I. Hennins, T. R. Jarboe and G. J. Marklin, Phys. Rev. Letters, 65, 40 (1990).

3. J. B. Taylor, Phys. Rev. Letters, 33, 1139 (1974); Rev. Mod. Phys. 58, 341 (1986).

4. F. J. Wysocki, J. C. Fernandez, I. Hennins, T. R. Jarboe, and E. J. Marklin, Phys. Rev. Letters, 61, 2457 (1988).

5. A. Bhattacharjee and E. Hameiri, Phys. Rev. Letters, 57, 206 (1986).

6. A. B. Rechester and M. N. Rosenbluth, Phys. Rev. Letters, 40, 38 (1978).

7. H. R. Strauss, Phys. Fluids, 29, 3668 (1986).

8. J. W. Connor and J. B. Taylor, Phys. Fluids, 27, 2676 (1984).

9. R. J. Lahaye, T. N. Carlstrom, R. R. Goforth, G. L. Jackson, M. J. Schaffer, T. Tamano, and P. . Taylor, Phys. Fluids, 27, 2756 (1984).

10. See also E. B. Hooper [Editor: please supply reference to Hooper, et al., paper from the Oak Ridge Conference, July (1994) appearing in this same volume of Fusion Technology].

11. R. Krakowski [Editor: please supply a reference to Krakowski's paper (from the Oak Ridge Conference, July 1994) appearing in this same volume of Fusion Technology].

12. T. K. Fowler and W. E. Drummond, Phys. Fluids, 10, 1110 (1967). 


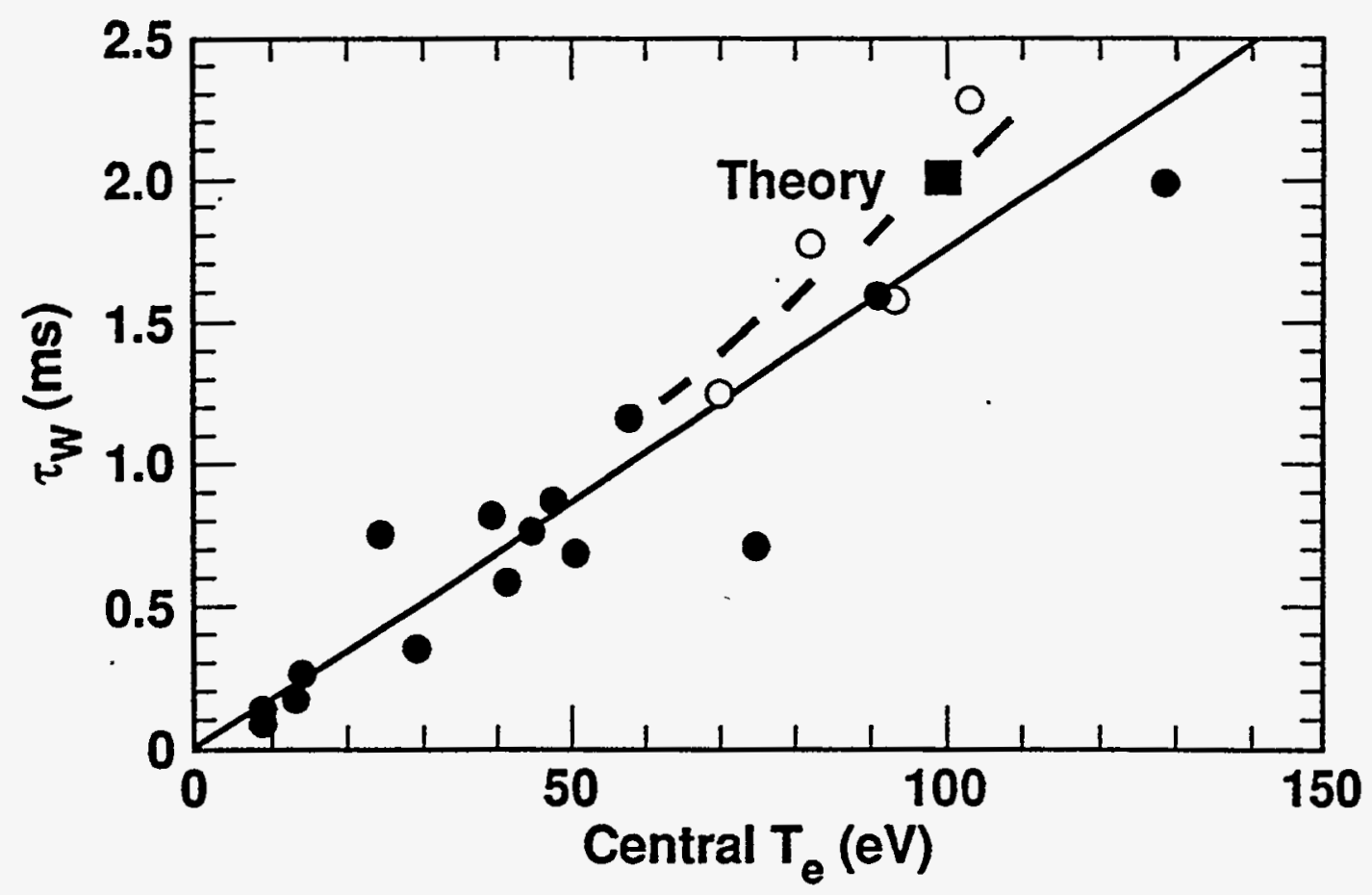

Fig.1. Magnetic decay time in CTX. From Ref. 2.

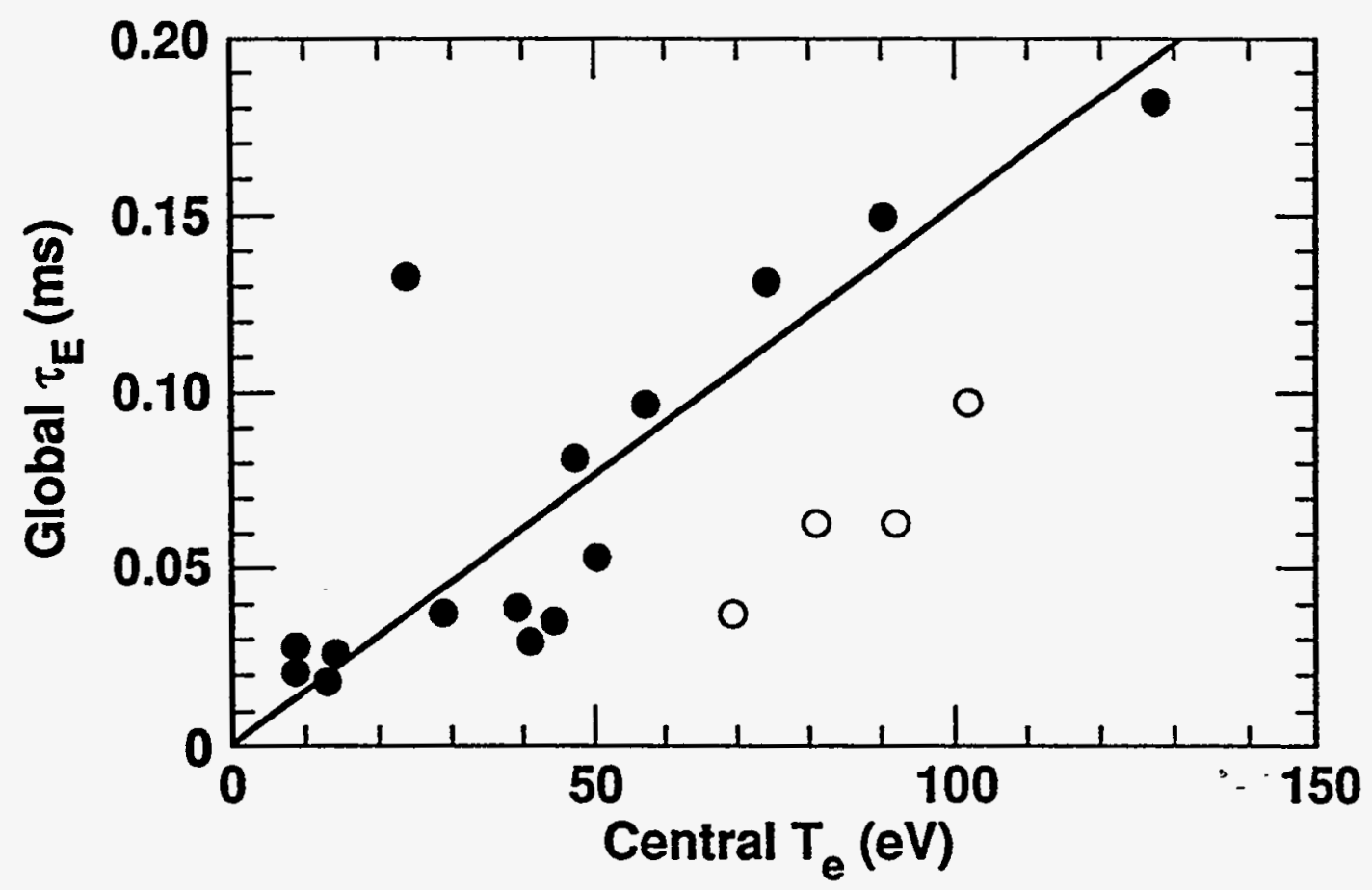

Fig. 2. Global energy confinement time in CTX. From Ref. 2. 